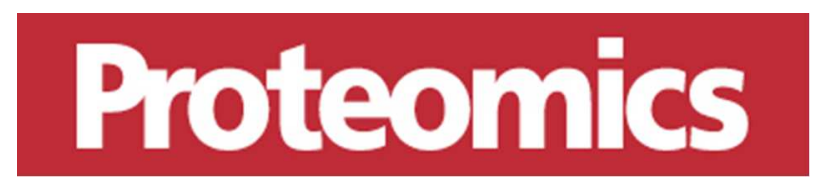

\title{
Contribution of extracellular matrix and signal mechanotransduction to epithelial cell damage in Inflammatory Bowel Disease patients: a proteomic study.
}

\begin{tabular}{|c|c|}
\hline Journal: & PROTEOMICS \\
\hline Manuscript ID & Draft \\
\hline Wiley - Manuscript type: & Research Article \\
\hline Date Submitted by the Author: & $\mathrm{n} / \mathrm{a}$ \\
\hline Complete List of Authors: & $\begin{array}{l}\text { Moriggi, Manuela; University of Milano, Italy, Department of Biomedical } \\
\text { Sciences for Health } \\
\text { Pastorelli, Luca; University of Milano, Italy, Department of Biomedical } \\
\text { Sciences for Health; IRCCS Policlinico San Donato, Gastroenterology and } \\
\text { Digestive Endoscopy Unit } \\
\text { Torretta, Enrica; University of Milano, Italy, Department of Biomedical } \\
\text { Sciences for Health } \\
\text { Tontini, Gian Eugenio; IRCCS Policlinico San Donato, Gastroenterology and } \\
\text { Digestive Endoscopy Unit } \\
\text { Capitanio, Daniele; University of Milano, Italy, Department of Biomedical } \\
\text { Sciences for Health } \\
\text { Ferrero Bogetto, Stefano; Ospedale Maggiore Policlinico, Biomedical, } \\
\text { Surgical and Dental Sciences } \\
\text { Vecchi, Maurizio; University of Milano, Italy, Department of Biomedical } \\
\text { Sciences for Health; IRCCS Policlinico San Donato, Gastroenterology and } \\
\text { Digestive Endoscopy Unit } \\
\text { Gelfi, Cecilia; University of Milano, Italy, Department of Biomedical } \\
\text { Sciences for Health; IRCCS Policlinico San Donato, Clinical Proteomics Unit }\end{array}$ \\
\hline Keywords: & $\begin{array}{l}\text { autophagy, extracellular matrix, inflammatory bowel diseases, } \\
\text { mechanotransduction }\end{array}$ \\
\hline
\end{tabular}




\title{
Contribution of extracellular matrix and signal mechanotransduction to epithelial cell damage in Inflammatory Bowel Disease patients: a proteomic study
}

\author{
Manuela Moriggi ${ }^{1}$, Luca Pastorelli ${ }^{1,2}$, Enrica Torretta ${ }^{1}$, Gian Eugenio Tontini ${ }^{2}$, Daniele Capitanio ${ }^{1}$, Stefano \\ Ferrero Bogetto $^{3}$, Maurizio Vecchi ${ }^{1,2}$, Cecilia Gelfi ${ }^{1,4}$ \\ ${ }^{1}$ Department of Biomedical Sciences for Health, University of Milan, Milan, Italy \\ ${ }^{2}$ Gastroenterology and Digestive Endoscopy Unit, IRCCS Policlinico San Donato, San Donato Milanese, \\ Italy \\ ${ }^{3}$ Biomedical, Surgical and Dental Sciences, IRCCS Ospedale Maggiore Policlinico \\ ${ }^{4}$ Clinical Proteomics Unit, IRCCS Policlinico San Donato, San Donato Milanese (MI), Italy \\ Corresponding author: Professor Cecilia Gelfi, \\ Department of Biomedical Sciences for Health, University of Milan, Via F.1li Cervi 93, 20090, Segrate (MI), \\ Italy \\ E-mail: cecilia.gelfi@unimi.it \\ Fax: $+39(02) 21717558$
}
Abbreviations:
BVA, biological variation analysis
C, control
$\mathrm{CD}$, Crohn's disease
DIA, differential in-gel analysis
ECM, extracellular matrix
EDA, extended data analysis
FDR, false discovery rate
GWAS, genome-wide association study
IBD, inflammatory bowel diseases
ICD, inflamed CD
ICPL, isotope-coded protein labeling
IEC, intestinal epithelial cells
IUC, inflamed UC
QCD, non-inflamed CD
QUC, non-inflamed UC
$\mathrm{UC}$, ulcerative colitis 
vs, versus

Keywords: autophagy, extracellular matrix, inflammatory bowel diseases, mechanotransduction

Total number of words: 10011

8

10

11

12

13

14

15

16

17

18

19

20

21

22

23

24

25

26

27

28

29

30

31

32

33

34

35

36

37

38

39

40

41

42

43

44

45

46

47

48

49

50

51

52

53

54

55

56

57

58

59

60 


\begin{abstract}
This study utilized 2D-DIGE, isotope-coded protein labelling (ICPL) and biochemical assays to characterize protein alteration in Ulcerative Colitis (UC) and Crohn's Disease (CD) in human epithelial cell and mucosal biopsies in Inflammatory Bowel Disease (IBD)-affected patients. The aim of this study is to identify the key molecular signatures involved in epithelial cell structure of IBDs.

In non-inflamed UC (QUC) keratins, vimentin and FAK increased, whereas vinculin and de-tyrosinated $\alpha$ tubulin decreased; inflammation (IUC) exacerbated molecular changes, being COL6A1, tenascin-C and vimentin increased. In non-inflamed CD (QCD) TNC, de-tyrosinated $\alpha$-tubulin, vinculin, FAK and ROCK1 decreased while vimentin increased; in inflamed CD (ICD) COL6A1 and vimentin increased. In QUC, cell metabolism was characterized by a decrease of the TCA cycle enzymes and a decrease of ACADSB, FASN, ppara and ppary. In QCD a metabolic rewiring occurred, as suggested by GPD2, PDHB, NDUFS3 and ALDH9A1 increment, while DLD decreased. Macroautophagy was activated in QUC and IUC, with increased levels of p62, HSC70, MVP, MYH9, whereas it was blunted in QCD and ICD.

The differing pattern of ECM, cytoskeletal derangements, cellular metabolism and autophagy in UC and CD may contribute to the pathophysiological understanding of these disorders and serve as diagnostic markers in IBD patients.
\end{abstract}




\section{Statement of significance of the study}

The identification of potential disease molecular signatures in UC and CD help shed light onto these complex disorders, highlighting new molecules, at cytoskeletal, metabolic and cell renewal level, which have never been described before in IBD. The study provides clear indication on the cytoskeletal rearrangement taking place in UC with the contribution of ECM and microenvironment to cell signalling from outside to inside the epithelial cell. Inflammation exacerbates this rearrangement, modifying membrane stiffness and favouring leucocytes homing. For the first time in IBD, the authors have described the increment of a specific enzyme involved in isoleucine catabolism with activity towards the degradation of branched-chain fatty acids (HSD17B10) that contribute to cell homeostasis and make cells able to support energy requirements by promoting the activation of the mTOR signalling. Another original observation is that in UC, macroautophagy is sustained by over-expression of p62, MVP and MYH9. Concerning $\mathrm{CD}$, its severity is highlighted by the absence of autophagy and cell renewal, whereas a metabolic rewiring to sustain cell metabolism occurred. Another unique aspect is the increase of vimentin, which can promote, together with impaired autophagy and an increase in COLVI, myofibroblasts transformation leading to fibrosis, a condition that characterises CD patients. 


\section{Introduction}

Inflammatory bowel diseases (IBD) are chronic and relapsing inflammatory conditions of the gastrointestinal tract. They comprise Crohn's Disease (CD) and Ulcerative Colitis (UC). Both CD and UC are characterized by a persistent inflammation that disrupts both the structure and functioning of the gastrointestinal mucosa. The aetiology and exact pathogenic mechanisms of IBD are unknown, however much interest has recently focused on an aberrant immune-inflammatory response to intestinal microbiota in genetically predisposed individuals. An important role in the interaction between microbiota and external antigens with our organism is attributed to the intestinal epithelium, which is no longer identified as a mere effector of absorptive and secretory activities but is recognized as an immunologically active cell population as well as a critical barrier to the penetration of threatening external factors. Normally, the intestinal epithelium is covered by a single layer of intestinal epithelial cells (IECs), which are characterised by a fast renewal rate, and it acts as a protective barrier against luminal antigens. Cells are linked by intercellular tight junctions, located at the apical ends of the lateral membrane, and they are coated by a thick adherent mucus gel, which forms a dynamic physical interface [1]. This barrier represents the largest area of contact between the inside of our body and the external environment and it is surrounded by many inflammatory cell types. Despite its robust and multi-faceted nature, this barrier can be damaged, thus promoting a state of chronic inflammation due to mucosal immune cell infiltration, as is typically observed in IBD patients [2]. Indeed, at least for UC, the important role of intestinal epithelial dysfunction has been suggested and the possible role of intestinal epithelium-derived antigens as the autoantigen has been proposed [3]. More recently, several genome-wide association studies (GWAS) have identified the intestinal epithelium as an important actor in the pathogenesis of IBD, indicating several genes related to the epithelial structure. The mechanism of autophagy may also be a possible contributor to $\operatorname{IBD}$ predisposition $[4,5]$. Indeed, the presence of specific polymorphisms in the following genes has been shown to be associated with the development of IBD: Nucleotide- binding oligomerisation domain-containing protein2 (NOD2) [6, 7], Autophagy-related protein 16-like 1 (ATG16L1) [8], Unc-51 like autophagy activating kinase 1 (ULK1) [9], Extracellular matrix protein 1 (ECM1), Tubulin polymerization-promoting protein (TPP1), Collagen Type VII Alpha 1 Chain (COL7A1) [10], ADAM Metallopeptidase Domain 17 (ADAM17) [11] and Fermitin Family Member 1 
(FERMT1) [12].

The typical activation of the inflammatory pathways and the consequent unbalance between pro- and antiinflammatory cytokines and mediators have been discussed in IBD for a long time; on the other hand, molecular changes of the epithelial tissue, including extracellular matrix and junction proteins in both inflamed and non-inflamed regions of the intestinal tract in IBD, have only partially been addressed. Thus, it appears that studies focusing on intestinal epithelium are warranted. In this respect, the classic proteomic approach has been frequently fruitful in the study of several other diseases of unknown aetiology and of possible immune-mediated pathogenesis. Thus far, proteomic studies on IBD patients have been mainly focused on the effects of inflammation rather than on the understanding of molecular mechanisms leading to disease onset or disease exacerbation. Overall, these studies have provided hints on the association of inflammation, energy metabolism and oxidative stress as possible driving forces for disease onset and exacerbation [13-17].

Recent independent studies by our group have highlighted the relationship between ECM dysregulation caused by Collagen VI deficiency and signal transmission from outside to inside the muscle cell. This signalling impacts on mitochondrial homeostasis and autophagy leading to muscle lipotoxicity [18, 19]. In the present paper, in order to address the above issues on intestinal epithelial cells from IBD patients, an unbiased "omics" approach was carried out to possibly unravel the physiopathological mechanisms that may be involved in intestinal epithelial cells homeostasis disruption in UC and CD patients and to detect selective changes that are able to differentiate between $\mathrm{CD}$ and $\mathrm{UC}$.

\section{Materials and methods}

\subsection{Sample collection}

\subsubsection{Ethics statement}

The Internal Review Board of the local Ethical Committee approved the study (Ethical Committee Protocol number n. 2726, ASL Milano-2, approved on October $2^{\text {nd }} 2012$ ), in accordance with the 1964 Declaration of Helsinki.

\subsubsection{Patients}


Colonic surgical specimens and non-inflammatory controls were obtained from either UC- or CD-affected male and female patients, aged 21 to 82 years, who were admitted to the IRCCS Policlinico San Donato (San Donato Milanese, Italy) and who underwent therapeutic bowel resection for IBD or for malignant and other non-malignant non-inflammatory conditions. Full-thickness colonic tissues were used as a source for the mucosal IEC. In patients who underwent surgical resection for colonic neoplasia, the part of surgical specimen that was collected and used for IEC isolation was macroscopically normal and was the most distant from the neoplasia.

Colonic endoscopic biopsies and controls were harvested from male and female patients affected by CD or UC, aged 25-70 years, who underwent colonoscopy for diagnostic or surveillance purposes at the IRCCS Policlinico San Donato; these were used for protein extraction.

\subsubsection{Isolation of Intestinal Epithelial Cells from surgical specimens}

Colonic surgical specimens were collected and processed immediately after surgical resection, as previously described [20]. Briefly, specimens were opened longitudinally, rinsed and examined for macroscopic morphological alterations, and representative full-thickness samples were obtained. Intestinal mucosa was stripped from the muscularis mucosa, cut into strips and washed in Hank's Balanced Salt Solution (HBSS; Invitrogen/GIBCO) containing $10 \mathrm{mM}$ DTT (Sigma-Aldrich) in order to remove mucus. IEC were isolated by repeated incubations in HBSS containing 1 mM EDTA (Sigma-Aldrich). A 40\% Percoll Plus gradient (GE Healthcare), where the IEC was equilibrated at the interface, was used in order to remove mononuclear, red blood and dead cells; resulting preparations enriched for IEC were collected and washed twice in PBS, and subsequently counted. Cell preparations were stained by means of an immunoperoxidase method, with an anti-keratin $\mathrm{mAb}$ and a monoclonal antibody directed against a leukocyte common $\mathrm{Ag}(\mathrm{CD} 45)$, resulting in a $<1 \%$ contamination of epithelial cell preparations by LPMC.

\subsection{Proteomic analysis}

Intestinal epithelial cell extracts from surgical specimens were obtained from patients with inflamed UC and CD (IUC, ICD; n=10) and patients with non-inflamed UC and CD (QUC, QCD; n=10) and from normal pathological specimens obtained from individuals without IBD (control, $C ; n=10$ ); these were analysed by 
2D-DIGE, isotope-coded protein labeling (ICPL) and immunoblot assay to evaluate proteome changes in inflamed and non-inflamed UC and CD patients compared to healthy controls.

Confirmatory blots were performed on biopsy specimens from inflamed and non-inflamed colonic mucosa obtained during colonoscopy by means of endoscopic biopsies from IUC, QUC, ICD and QCD patients $(n=5 /$ experimental group) and controls $(n=6)$.

\subsubsection{Protein extraction}

For 2D-DIGE and immunoblot assays, each sample from each subject was suspended in lysis buffer (7 M urea, $2 \mathrm{M}$ thiourea, 4\% CHAPS, $30 \mathrm{mM}$ Tris, and $1 \mathrm{mM}$ PMSF) and solubilized by sonication on ice. Proteins were selectively precipitated using PlusOne 2D-Clean up Kit (GE Healthcare, Little Chalfont, UK) in order to remove non-protein impurities, and they were re-suspended in lysis buffer. The $\mathrm{pH}$ of the protein extracts was adjusted to $\mathrm{pH} 8.5$ by addition of $1 \mathrm{M} \mathrm{NaOH}$. Protein concentrations were determined by PlusOne 2D-Quant Kit (GE Healthcare).

\subsubsection{D-DIGE}

Intestinal epithelial cells extracts were analysed by quantitative 2D-DIGE, followed by mass spectrometry. The 2D-DIGE method was inserted in a MIAPE-GE compliant form [21] in Supporting Information Table 1S. Protein minimal labelling with cyanine dyes (Cy3 and Cy5), 2D separation and analyses were performed as described previously [22]. Briefly, the proteins extracted $(50 \mu \mathrm{g})$ from each individual sample were labelled with Cy5, while internal standards were generated by pooling $(50 \mu \mathrm{g})$ individual samples that were Cy3-labeled. Samples were separated on 3-10 nonlinear immobilized pH gradient (IPG) strips; each individual sample was run in triplicate (analytical replicates) to minimize the inter-gel variability and increase the reliability of the results. Image analysis was performed using DeCyder 6.5 software (GE Healthcare). All gel images were imported into individual differential in-gel analysis (DIA) workspaces. Using the Batch Processor tool, automated detection of protein spots was performed with the following filter settings: estimated number of spots $=10000$, exclusion slope $>1.2$; minimal area cutoff $<200$, and peak height $=100000$. DIA workspaces were then manually edited to eliminate gel artefacts (e.g., plate scratches and dust specks) and include any incorrectly excluded spot. The resulting spot maps (containing the spot identifiers, locations and normalized volumes for all protein spots in each channel of each gel) were further 
processed in the biological variation analysis (BVA) module. Individual DIA workspaces for all analytical gels were imported into the BVA module. The BVA workspace was used for inter-gel protein spot matching. Statistical analysis was performed using the DeCyder 1.0 extended data analysis (EDA) module. Protein filters were set to select only those protein spots that matched $90 \%$ of the gel images and these protein spots were included in data analysis. For proteomic experiments, statistically significant differences were computed by independent one-way ANOVA, coupled to Turkey's multiple group comparison test; the significance level was set at $\mathrm{p}<0.01$. To minimize inclusion of false-positive protein spot changes, protein expression data were filtered using the following criteria: independent one-way ANOVA, coupled to Turkey's multiple group comparison test $(\mathrm{p}<0.01), 1.15$-fold difference in abundance, and false discovery rate (FDR). The change of 1.15-fold and above in protein abundance was adopted for the present analysis, taking into account the power of the DIGE method to detect reliable differences in protein abundance down to $15 \%[23,24]$. FDR correction was applied as a multiple testing correction method to keep the overall error rate as low as possible [25]. Proteins of interest were identified by mass spectrometry.

\subsubsection{Protein identification by MALDI-TOF MS}

Semipreparative gels, containing $400 \mu \mathrm{g}$ of total protein extract per strip, were loaded; electrophoretic conditions were the same as for 2D-DIGE, except that gels were stained with a protein fluorescent stain (Deep Purple Total Protein Stain, GE Healthcare). Image acquisition was performed using the Typhoon 9200 laser scanner. Proteins were identified by PMF utilizing a MALDI-ToF mass spectrometer (Ultraflex III ToF/ToF; Bruker Daltonics, Bremen, Germany), as previously described [26]. In particular, the search was performed by correlation of uninterpreted spectra to Homo sapiens entries in NCBInr database 20100918 (11833178 sequences; 4040378175 residues). Protein identification methods are provided in a MIAPE-MS compliant form [21] in Supporting Information Table 1S. (For further information about protein identification, see Supporting Information Table 2S and Supporting Information Figure 1S).

\subsubsection{ICPL}

Intestinal epithelial cell extracts obtained from QUC, QCD and C were analysed by differential proteomics based on ICPL. A total of $100 \mu \mathrm{g}$ of proteins per sample were purified using the PlusOne 2D-Clean up Kit (GE Healthcare) and protein pellets were then dissolved in $6 \mathrm{M}$ guanidine $\mathrm{HCl}, \mathrm{pH}$ 8.5. Protein 
concentration was determined using the PlusOne 2D-Quant Kit (GE Healthcare) and adjusted to 5-10 $\mu \mathrm{g} / \mu \mathrm{L}$ in all samples. ICPL labelling of proteins was performed according to the manufacturer's instructions using the SERVA ICPL ${ }^{\mathrm{TM}}$ Quadruplex PLUS Kit (Serva, Heidelberg, Germany). C, QUC and QCD were labelled using light ICPL_0, heavy ICPL_6 and ICPL_10 labeled reagents, respectively. The three labeled samples were then combined and the proteins were purified by acetone precipitation at $-20^{\circ} \mathrm{C}$ overnight. The mixture was centrifuged at $20000 \times \mathrm{g}$ for $30 \mathrm{~min}$ at $+4^{\circ} \mathrm{C}$. The supernatant was discarded and remaining acetone was removed by evaporation at RT. The protein pellet was then dissolved in $50 \mathrm{mM}$ ammonium bicarbonate and RapiGest SF surfactant (Waters Corporation) and protein concentration was determined using the PlusOne 2D-Quant Kit. Reduction and alkylation were performed after adjusting the $\mathrm{pH}$ of each sample to 8.5 , and using DTT (incubation at $60^{\circ} \mathrm{C}$ for $30 \mathrm{~min}$ ) and iodoacetamide (incubation at RT in the dark for $30 \mathrm{~min}$ ), respectively. Finally, proteins were digested using trypsin at an enzyme/substrate ratio of 1:50 overnight at $37^{\circ} \mathrm{C}$. Digestion was stopped by formic acid $0.1 \%$ (v/v, final concentration). The resulting peptides were separated by reverse phase chromatography $(75 \mu \mathrm{m}$ id x15cm PepMap column, Thermo Fisher Scientific) using the nano-HPLC EASY-nLC (Bruker Daltonics) through a linear gradient of 85 minutes from $5 \%$ B to $60 \%$ B (solvent A: water/0.05 \% TFA, solvent B: 90\% ACN/9.95\% water/0.05\% TFA) with a flow of 300 $\mathrm{nL} / \mathrm{min}$. The $\mathrm{n}-\mathrm{LC}$ was coupled with the high-resolution mass spectrometer MaXis hybrid UHR-QToF (Bruker Daltonics) for protein identification. Protein quantification was performed on the basis of peptides, by using Warp-LC v. 1.2 (Bruker Daltonics). The extracted ion chromatograms (EICs) were calculated by using the $\mathrm{m} / \mathrm{z}$ values of identified peptides and summing the intensities of the mass peaks (mass tolerance of 0.5 Da). A retention time window was fixed at $0.4 \mathrm{~min}$ around the elution time of the identified peptides, and only charge states $+2,+3$ and +4 were taken into account when the EIC was generated. The chromatographic peak finder in Data Analysis (Bruker Daltonics) was adopted to detect peaks, and only peptides with an ion score above 20 were used to quantify protein species. The areas under the curve were calculated by Data Analysis chromatographic peak finder; of the first three peaks of the isotopic distribution were summed for both the heavy and light components of an isotopic pair. The regulation ratio was calculated using the summed intensities. For statistical analysis, all data were converted in the log space to maintain symmetry around zero. The global mean and SD of protein ratios were calculated for each couple of the sample. The 
cut-off point for protein differential abundance was determined as the mean $\pm 2 \mathrm{SD}(95 \%$ confidence interval) and protein differential abundance was assessed using the Student's t-test. (For further information about protein identification, see Supporting Information Table 3S)

\subsubsection{D-DIGE and ICPL orthogonal validation by immunoblotting}

The 2D-DIGE and ICPL results were validated performing a random analysis via immunoblotting of $16 \%$ of the identified proteins (Supporting Information Figure 2). Protein extracts $(50 \mu \mathrm{g})$ from pooled samples were loaded in triplicate and resolved on 6-14\% polyacrylamide gels. Blots were incubated with rabbit, mouse or goat polyclonal primary antibodies as follows: anti-keratin type I cytoskeletal 19 (KRT19; GeneTex, CA, USA), 1:3000; anti-tubulin beta-2C chain (TUBB4B; Sigma Aldrich, USA), 1:1000; anti-succinate dehydrogenase [ubiquinone] flavoprotein subunit, mitochondrial (SDHA; Santa CruzBiotechnology, CA, USA), 1:1000; anti-galectin-3 (LGALS3; Abnova, Taipei City, Taiwan), 1:5000; anti-prohibitin (PHB, GeneTex), 1:5000; anti-keratin type I cytoskeletal 18 (KRT18; Cell Signaling Technology, Danvers, MA, USA), 1:2000; anti-heat shock cognate 71 kDa protein (HSC70; Cell Signaling Technology), 1:1000; anti78 kDa glucose-regulated protein (GRP78; Santa Cruz Biotechnology), 1:500.

After washing, membranes were incubated with anti-rabbit or anti-mouse (GE Healthcare, Milan, Italy) or anti-goat (Santa Cruz Biotechnology) secondary antibodies, conjugated with horseradish peroxidase. Signals were visualized by chemiluminescence using the ECL Prime detection kit (GE Healthcare). Image analysis (Image Quant TL, Molecular Dynamics, Ragusa, Italy) was performed, followed by statistical analysis (Student's t-test, $\mathrm{p}<0.05$ ). Data were normalized against the total amount of loaded proteins stained with Sypro Ruby Blot Stain (Life Technologies Europe BV, Monza, Italy).

\subsection{Protein quantitation by immunoblotting}

Immunoblotting procedures were as described above. Blots were incubated with rabbit, mouse or goat polyclonal primary antibodies (Santa Cruz Biotechnology, except when differently stated) as follows: anticollagen type VI alpha 1 chain (COL6A1), 1:200; anti-tenascin C (TNC), 1:1000; anti-integrin $\beta 7,1: 500$; anti-integrin $\alpha 4$, 1:500; anti-focal adhesion kinase (FAK; Cell Signaling Technology), 1:1000; anti-Rhoassociated protein kinase 1 (ROCK1; Cell Signaling Technology), 1:1000; anti-vimentin, 1:1000; antidetyrosinated $\alpha$-tubulin (de Tyr $\alpha$-Tubulin; Abcam, Cambridge, UK), 1:500; anti-vinculin, 1:500; anti-citrate 
synthase, 1:500; anti-fatty acid synthase (FASN), 1:100; anti-peroxisome proliferator-activated receptors gamma (ppary), 1:500; anti-peroxisome proliferator-activated receptors alpha (ppar $\alpha$ ), 1:500; anti-heat shock cognate 71 kDa protein (HSC70), 1:1000; anti-myosin heavy chain 9 (MYH9), 1:500; anti-major vault protein (MVP); 1:2000; anti-ubiquitin-binding protein p62 (p62; Sigma Aldrich), 1:1000; anti-light chain 3B (LC3B; Cell Signaling Technology), 1:500; anti-bcl-2-like protein 4 (BAX), 1:1000; anti-beclin1, 1:1000; anti-B-cell lymphoma-extra large (Bcl-xL; Cell Signaling Technology), 1:1000; anti-BCL2/adenovirus E1B 19 kDa protein-interacting protein 3 (Bnip3) 1:1000; B-cell leukemia/lymphoma 2 (Bcl-2; Cell Signaling Technology).

\section{Results}

\subsection{Comparative proteomic analysis}

To unravel possible molecular processes involved in disease aetiology, intestinal epithelial cell extracts from UC, CD patients and controls were analysed by 2D-DIGE, ICPL LC MS/MS and immunoblot assay.

\subsubsection{D-DIGE}

Intestinal epithelial cell extracts were analysed by 2D-DIGE to assess proteome changes in non-inflamed $\mathrm{UC}$ and CD patients, compared to controls.

Concerning UC, epithelial cells were purified from surgical specimens from 10 patients with non-inflamed

UC and 10 controls. Overall, 1000 spots were matched among gels and 22 spots were changed in abundance in QUC vs C. With regard to CD, epithelial cells obtained from patients with non-inflamed CD ( $\mathrm{n}=10)$ and individuals without obvious inflammatory colonic disorder $(n=10)$ were purified as previously described [20]. The proteomic analysis provided 27 spots changes in abundance in QCD vs C. A representative 2D map of intestinal epithelial cells, after cell extract separation in 3-10 non linear $\mathrm{pH}$ gradient IPG strips, is shown in Supporting Information Figure 3S. The entire list of proteins differentially expressed together with statistical analyses, protein accession number, gene name, theoretical molecular mass, isoelectric points and MS data are listed in Supporting Information Table 2S. The protein identification was validated performing a random analysis via immunoblotting of $16 \%$ of the identified proteins (see Supporting Information Figure $2 \mathrm{~S})$. The identified proteins were then grouped according to their role. 
The semiquantitative proteomic profile of QUC and QCD vs C (Figure 1) revealed a peculiar signature for $\mathrm{UC}$ and $\mathrm{CD}$, in which changes of proteins involved in cytoskeleton organization, metabolism, stress and antioxidant response played a prominent role.

Cytoskeletal dysregulation in QUC vs C was characterized by a decrease in keratin type II cytoskeletal 8 (KRT8) [27], actin cytoplasmic 1 (ACTB), tubulin beta-2C chain (TUBB4B) and mitochondrial inner membrane protein (IMMT), whereas keratin type I cytoskeletal 19 (KRT19) increased. Several metabolic proteins were decreased, such as: succinate dehydrogenase [ubiquinone] flavoprotein subunit (SDHA), ATP synthase subunit beta (ATP5B) and short/branched chain specific acyl-CoA dehydrogenase (ACADSB). The latter catalyses the dehydrogenation of acyl-CoA derivatives and is involved in L-leucin catabolism. Conversely, there was an increase in 3-hydroxyacyl-CoA dehydrogenase type-2 (HSD17B10), which catalyses the oxidation of neuroactive steroids and represents the third step in the beta oxidation of fatty acids [28]. Among the proteins involved in stress response: $60 \mathrm{kDa}$ heat shock protein (HSP60), preventing protein misfolding) and peroxiredoxin-2 (PRDX2) both decreased, whereas the protein disulfide-isomerase A3 (GRP58) was more abundant in QUC than in C. The Elongation factor tu (TUFM), which regulates protein synthesis, was down-regulated in QUC compared to controls.

Conversely, QCD vs C was characterized by a down-regulation of KRT8 and keratin type I cytoskeletal 18 (KRT18). Metabolic proteins of QCD vs C showed a down-regulation of dihydrolipoyl dehydrogenase (DLD), whereas the authors observed an up-regulation of glycerol-3-phosphate dehydrogenase (GPD2), pyruvate dehydrogenase E1 component subunit beta (PDHB), NADH dehydrogenase [ubiquinone] ironsulfur protein 3 (NDUFS3) and 4-trimethylaminobutyraldehyde dehydrogenase (ALDH9A1), involved in the pathway of carnitine and polyamine biosynthesis. Concerning stress response proteins: heat shock cognate $71 \mathrm{kDa}$ protein (HSC70), which is involved in chaperone-mediated autophagy; heat shock $70 \mathrm{kDa}$ protein 1A/1B (HSPA1A/B), which contributes to the homeostasis of the immune system by directly modulating the expansion and function of conventional T-cells; $78 \mathrm{kDa}$ glucose-regulated protein (GRP78), GRP58 and PRDX2 were decreased, thus suggesting the activation of endothelial reticulum (ER) stress and unfolded protein response (UPR). Annexin A4 (ANXA4) and annexin A5 (ANXA5), which bind negatively charged phospholipids in a calcium dependent manner, were less abundant in QCD, compared to controls. 
Voltage-dependent anion-selective channel protein 1 (VDAC1), the mitochondrial 28S ribosomal protein S22 (MRPS22), the endoplasmic reticulum lipid raft-associated protein 2 (ERLIN2), which mediates the endoplasmic reticulum-associated sterol degradation (ERAD), and the complement component $1 \mathrm{Q}$ subcomponent-binding protein (C1QBP) involved in inflammation and infection processes, were upregulated in QCD.

Both QUC and QCD compared to C were characterized by a down-regulation of prohibitin. The mitochondrial prohibitin complex controls cell proliferation, cristae morphogenesis and the functional integrity of mitochondria and inhibits DNA synthesis. Galectin-3 (LGALS3) and carbonic anhydrase 1 (CA1) were also decreased. Conversely, the receptor of activated protein C kinase 1 (RACK1, shuttling proteins around the cell and anchoring proteins at particular locations stabilising protein activity) was upregulated, compared to controls [29].

\subsubsection{ICPL}

To increase the data set of proteomic changes in UC and CD epithelial cell extracts, an ICPL analysis was performed (Figure 2). This quantitative proteomic approach allowed for the identification and quantification of 2648 peptides, corresponding to 1058 proteins. A stringent statistical analysis was applied to this set of identified proteins and only proteins identified by 5 peptides and common to all analysed samples were considered. Collectively, 19 proteins were dysregulated in QUC compared to controls, 18 increased and one decreased, whereas 5 were at variance in QCD vs $\mathrm{C}$ (2 increased and 3 decreased). The first observation is that the set of dysregulated proteins is different from the group recognized by 2D-DIGE, but few proteins were common to both proteomic analyses, confirming the relevance of these two independent proteomic approaches.

QUC compared to C is characterized by increased levels of structural proteins like myosin-9 (MYH9), keratin type I cytoskeletal 10 (KRT10), keratin type I cytoskeletal 14 (KRT14), KRT18, tubulin alphaubiquitous chain (TUBA1B) and vimentin (VIM). On the contrary, the corticosteroid 11-betadehydrogenase isozyme 2 (HSD11B2), which is related to the transformation of cortisol into cortisone to counteract inflammation by increasing glucocorticoids, was significantly decreased. Proteins involved in transcription regulation and folding were also increased, particularly, the heterogeneous nuclear 
ribonucleoprotein A1 (HNRPNA1), which is involved in the packaging of pre-mRNA into hnRNP particles and transport of poly(A) mRNA from the nucleus, and the major vault protein (MVP), which acts as scaffolds for proteins involved in signal transduction. A number of proteins playing a role in chromatin remodelling, like histone H2A type 2-A (HIST2H2AA 3), type 1-B (HIST1H2AB), type 1-C (HIST1H2AC), histone H3.1 (HIST1H3A) and histone H4 (HIST1H4A), which is a core components of the nucleosome, were increased. Proteins associated to protein folding and transport were increased, including: the splicing factor proline- and glutamine-rich (SFPQ), which is involved in transcriptional regulation and recruitment of histone deacetylases (HDACs); the heat shock protein $75 \mathrm{kDa}$ (TRAP1), which is involved in maintaining mitochondrial function and polarization most likely through stabilization of mitochondrial complex I; the peptidyl-prolyl cis-trans isomerase B precursor (PPIB), which catalyses the transcription of DNA into RNA; the ribosome-binding protein 1 (RRBP1), which mediates the interaction between the ribosome and the endoplasmic reticulum membrane; and coatomer subunit alpha (COPA), which is a cytosolic protein complex essential for the retrograde Golgi-to-ER transport of dilysine-tagged proteins.

Concerning QCD, this study confirmed a smaller and different spectrum of protein variations compared to QUC: sodium/potassium-transporting ATPase alpha-1 chain precursor (ATP1A1) and HIST1H3A increased, whereas MYH9, MVP and HIST1H2AC decreased in QCD compared to C.

From the present proteomic data, protein variation suggests that at epithelial cell level the disease impacts differently on the proteome signature of $\mathrm{UC}$ and $\mathrm{CD}$, though some features appear common. As a result, proteomic analysis offers a hint to decipher the pathophysiological mechanisms associated to diseases $[18,30]$, therefore further analysis to demonstrate the validity of proteomic data, not only through the validation of proteomic results by an independent technology but also assessing the impact of dysregulated protein on specific sub cellular compartments, could be relevant to gain better insight onto the pathophysiological mechanisms of these complex disorders.

\subsection{Protein quantitation by immunoblotting}

To further prove the role of cytoskeleton, metabolism and stress response dysregulations in the intestinal mucosa of UC and CD patients, quantitation of selected proteins and of some upstream regulators controlling their expression levels was evaluated by immunoblotting. 
These experiments focused on those cellular metabolic/structural pathways that were identified by previous proteomic analyses (e.g. cytoskeleton rearrangement, metabolism and stress response). Interestingly, these pathways can significantly impact cell signalling from inside to outside the epithelial cell and vice-versa, leading to cell death and failure of cell renewal.

In this experimental section, protein extracts from colonic endoscopic biopsies from inflamed and noninflamed regions of the gastrointestinal tract of $\mathrm{UC}$ and $\mathrm{CD}$ patients were analysed to explore the role of inflammation on the above-mentioned pathways in UC and CD.

\subsubsection{Cytoskeleton rearrangements: Stiffness and cell signal mechanotransduction}

Proteomic results indicated that the cytoskeletal components appear at variance in non-inflamed UC and CD compared to controls. In QUC versus controls, different proteoforms of KRT8, ACTB and TUBB4B were reduced, whereas KRT10, KRT14, KRT18, KRT19 and vimentin were increased. In QCD vs C, KRT8 and KRT18 were decreased. Collectively, these results suggest a possible remodelling of the cytoskeletal structure and an altered signal transmission from inside to outside or vice-versa of the epithelial barrier in $\mathrm{UC}$, whereas in CD changes appear modest, further supporting the need for upstream regulators of the mechanical signal transduction from outside to inside the epithelia cell to understand mechanisms associated to epithelial damage.

Figure 3 panel A and B shows the immunoblotting quantitative analysis of molecules involved in ECM molecular structure and signal mechanotransduction in QUC and IUC, compared to controls. With regards to QUC vs C, collagen VI alpha 1 chain (COL6A1) and tenascin C (TNC) were unchanged, whereas vinculin and detyrosinated $\alpha$-tubulin (de Tyr $\alpha$-Tubulin), localized on membrane surface, were reduced. Interestingly, focal adhesion kinase (FAK), which are the sensors inside the cell involved in cellular adhesion, and the structural protein vimentin were increased, whereas Rho-associated protein kinase 1 (ROCK1), which is involved in actin cytoskeleton organization and remodelling, decreased; this suggests a disconnection of the system. Inflammation of intestinal mucosa exacerbated molecular changes, being COL6A1, and TNC increased, whereas FAK decreased and ROCK1 increased, together with vimentin. Inflammation induces further cytoskeletal and ECM remodelling, which might have an impact on membrane mechanical properties. Concerning detyrosinated $\alpha$-tubulin and vinculin, these molecules were decreased similarly to non-inflamed 
IBD tissue, suggesting that these molecules are not targeted by inflammation and their expression changes are primary features of the IBD epithelial layer. To support the differential expression of proteins involved in signal mechanotransduction from outside to inside in inflamed tissues, and to determine whether lymphocytes' homing induced by inflammation can be associated to ECM and cytoskeletal protein dysregulation, integrin $\beta 7$ and $\alpha 4$, known to interact with the endothelial VCAM1 proteins [31, 32], were analysed by immunoblotting in colonic endoscopic biopsies. Results indicated that integrin $\beta 7$ and $\alpha 4$ were unchanged in QUC vs C, whereas they increased in IUC vs C, confirming the specific role of these molecules in inflammation [33].

Conversely (Figure 3 panel C and D), colonic endoscopic biopsies from QCD patients vs controls were characterized by normal levels of COL6A1 and integrin $\alpha 4$, whereas TNC, integrin $\beta 7$, detyrosinated $\alpha$ tubulin, vinculin, FAK and ROCK1 were decreased and vimentin was increased. In ICD vs controls, an increase of COL6A1, vimentin and integrin $\alpha 4$ was observed, whereas FAK, TNC, vinculin and detyrosinated $\alpha$-tubulin remained down-regulated, and ROCK1 and integrin $\beta 7$ remained unchanged. Overall, these data suggest the inability of epithelial cells to reorganize their structure under inflammatory conditions. These results also indicate that the response of ICD patients is at variance compared to IUC patients. In particular, what appears peculiar is the absence of signal response from outside to inside epithelia and, in this context, the increase of COL6A1 could be associated to integrin overexpression. Furthermore, the increase of vimentin is not related to inflammation response, but could be associated to the transformation of interstitial sub-epithelial fibroblasts into myofibroblasts contributing to fibrosis [34-36]. Further experiments are required to clearly demonstrate this hypothesis. Collectively, these results indicate that substantial differences in the molecules involved in epithelial molecular homeostasis and in inflammatory response are present between $\mathrm{UC}$ and $\mathrm{CD}$.

\subsubsection{Metabolism}

Proteomic results indicate dysregulation of enzymes involved in fatty acid oxidation (ACADSB and HSD17B10) and a decrease in two enzymes of oxidative phosphorylation (SDHA and ATP5B) in QUC vs C. QCD patients were characterized by an increase in GPD2, PDHB, NDUFS3 and ALDH9A1, whereas DLD enzyme appeared lower, suggesting that a metabolic rewiring could be adopted by the epithelial cell to 
sustain energy demand, involving polyamine synthesis finalized to citrate homeostasis maintenance.

According to the alteration of mechanotransduction signalling and to enzymes involved in oxidative energy production, the authors question whether citrate and fatty acids could be at variance, together with some upstream regulators, of the energy balance. Figure 4 shows citrate synthase, fatty acid synthase (FASN), peroxisome proliferator-activated receptors gamma (ppary) and alpha (ppara) assessed by antigen antibody reaction in QUC, IUC, QCD and ICD vs controls.

UC is characterized by an unchanged level of citrate synthase in non-inflamed patients, but this enzyme is decreased in IUC. Conversely, FASN, ppary and ppara were down-regulated both in QUC and IUC [37], suggesting a lipid synthesis and oxidation impairment not supported by alternative metabolic pathways.

Whereas the reduced expression/activation of ppary in UC has been repeatedly demonstrated, data on ppara appear at variance compared to recent results by Zhou X. et al., Nature Communication 2014, in chronic dextran sulphate sodium (DSS)-induced colitis mouse model, in which the genes involved in PPAR $\alpha$-UGTs signalling were increased. Further experiments will be required to precisely clarify this discrepancy between DSS-induced colitis mice and UC patients [38].

Concerning CD, decreased citrate levels could be associated to the dysregulation of specific metabolic enzymes, revealed by proteomics, suggesting a metabolic rewiring to compensate energy deficiency due to a decrement of lipid synthesis and oxidation characterizing non-inflamed epithelial CD cells. Under inflammation in $\mathrm{CD}$, the citrate synthase levels were normalized, whereas ppary increased. These results in QCD patients suggested a metabolic dysregulation at the alpha ketoglutarate metabolic node, which can induce a metabolic flux deviation toward polyamine and carnitine biosynthesis to refuel energy [39, 40].

\subsubsection{Autophagy in UC and CD}

Proteomic results indicate dysregulation of MYH9 and MVP proteins (for the first time described in IBDs), in both diseases and decrement of HSC70 and annexin 4 and 5 in QCD. Figure 5 shows levels of HSC70, MYH9 and MVP, respectively, in QUC and IUC compared to controls (panel A) and QCD and ICD compared to controls (panel B). The results indicate that HSC70 was unchanged in QUC while it increased in IUC. This result in UC cell extracts further supports the ability of these cells to activate the chaperonemediated autophagy. MYH9 only increased in QUC, while MVP increased in both QUC and IUC. 
Conversely, the MYH9 and MVP proteins were reduced in QCD and ICD, while HSC70 was downregulated in QCD and it remained unchanged in ICD, thus confirming the proteomic results. Collectively, these data suggest that, due to their involvement in cellular homeostasis, these proteins can influence the autophagic process. The role of MYH9 has been recently associated to cell renewal following the axis Myh9-Rac1-PAK1-Akt pathway in the maintenance of Lgr5+ stem cells in DSS induced colitis [41]. The same protein has also been recently associated to p62 levels [42].

Figure 5 panel D shows that p62 levels were increased in QUC and IUC compared to controls.

Conversely, p62 levels were decreased in QCD and were increased in ICD (Figure 5, panel F), even though in ICD the down regulation of LC3, of MYH9 and MVP indicates that, despite inflammation, rescue processes for eliminating damaged material are not efficiently activated.

To further clarify the nature of the autophagic process (macroautophagy vs chaperone-mediated autophagy) other molecules known to be involved in the autophagic flux regulation were assessed by immunoblotting. Autophagic flux was estimated by the autolysosome formation through LC3-II increase and p62 protein degradation or chaperone-mediated autophagy was assessed by the increase of the HSC70. Besides this, the molecules involved in autophagy activation/inhibition, such as BAX, beclin-1, Bcl-xL and BNIP3 were also considered. QUC was characterized by a decrease of BAX and BNIP3 and an increase of Beclin-1 and Bcl$\mathrm{xL}$, whereas Bcl2 was decreased though not enough to reach statistical significance. In IUC, the pattern is characterized by an increase in Bcl-xL and BNIP3 and a decrease in Bcl2.

Conversely, in QCD no changes were observed in BAX, Beclin-1 and Bcl2, whereas Bcl-xL was increased and BNIP 3 was decreased. ICD were characterized by increments of BAX, Bcl-xL and Bcl2, and a decreased of BNIP3, whereas Beclin-1 was unchanged [43].

\section{Discussion}

The unbiased differential proteomic approaches adopted in the present study provide evidence of several dysregulated molecules that characterise QUC and QCD epithelial cells. Collectively, these data suggest that ECM, mechanotransduction, metabolic rewiring and autophagy appear to be crucial nodes highlighting discrepancies and similarities in UC and CD at the molecular level and they may help shed light onto the physiopathological mechanisms characterizing these two disorders. It is worth noting that some results 
provided by this study have also been previously observed, both at genetic and protein level; while others are original and support the use of a global proteomic approach to investigate physiopathological mechanisms of a disease [13-17]. The major pitfall of this study is represented by the restricted number of samples analysed and the small, but still present, degree of contamination in our IEC preparation. Despite this, a rigorous methodological approach of analysis was used, since only proteins being differentially expressed in all samples were considered and, in the case of ICPL methodology, only proteins recognized by 5 peptides and with the same trend in all samples were taken into consideration. Finally, the validation step by antigen antibody reactions, in an independent set of samples and on mucosal biopsies was quite extensive, involving results from proteomic analyses and markers of upstream regulating pathways.

Given that, QUC vs C were characterized by a cytoskeletal rearrangement in which keratin 8 (which was recently described as an autoantigen in rheumatoid arthritis [27]) is decreased, whereas other keratins (KRT10, KRT14, KRT18, KRT19) are increased, thus suggesting a structural re-arrangement at the epithelial level. The latter can account for several pieces of evidence, indicating that physical modifications of the epithelial layers, leading to an impaired permeability and cell renewal, have a prominent role in UC pathogenesis $[44,45]$.

Indeed, IEC structural alterations parallel characteristic shifts in cell metabolic activity; in fact, in QUC vs controls, a decrease in enzymes of the TCA cycle and oxidative phosphorylation (succinate dehydrogenase and ATP synthase subunit beta), ACADSB, FASN, ppar $\alpha$ and ppar $\gamma$ was observed, suggesting a downregulation of oxidative metabolism and fatty acid synthesis. Unexpectedly, the proteomic analysis highlighted the increase of a specific mitochondrial enzyme involved in isoleucine catabolism, with activity towards the degradation of short branched-chain fatty acids (HSD17B10). This amino acid is both glucogenic and ketogenic, and under starvation, the reduced carbon skeleton is used for energy production. This metabolic route to produce acetyl-CoA is typical of QUC patients and could be adopted by cells to support energy requirements and promote the activation of mTOR signalling (see Supporting Information Figure 4S). On the other hand, GWAS data [5] are supportive of our results, indicating the role of the ULK1 gene in IBD. ULK1 is a tight regulator of TORC1/AMPK signalling pathway to sustain intracellular energy and nutrient levels in IBD counteracting starvation [9].Interestingly, in 1980, the hypothesis that UC may 
originate from a defect of fatty acid (n-butyrate) oxidation of IEC was first proposed [46]; along the same lines, topical treatment with butyrate enemas showed some efficacy in distal UC [47, 48]. The increase in HSD17B10 can suggest an adaptation of the epithelial cell to counteract energy deficiency. Furthermore, HSD17B10 is also essential for intracellular steroid metabolism, and altered HSD17B10 enzyme level levels may cause a dysregulation in steroid pathways affecting the inflammatory response of colonic mucosa [28]. The ECM components, such as COL6A1 and TNC, are involved in the integrity of the epithelial barrier and can influence outside to inside signalling, thus contributing to the lymphocytes homing. In this context, COLVI can promote the formation of the characteristic beaded microfilaments network in the ECM and act as a structural support and binding partner for other ECM proteins. Furthermore, it is involved in the inhibition of apoptosis and oxidative damage [49]. COL6A1 is a sensor of TGF- $\beta$ signalling $[50,51]$ and vinculin is the cross-talk between ECM and actin cytoskeleton for the mature focal adhesion assembly [52, 53]. FAK are necessary for the regulation of mechano-sensory activity of focal adhesions, and the small GTPase RhoA links the actin polymerisation at focal adhesions to the global actin dynamics [54]; FAK activation leads to the recruitment of PI3 kinase to focal adhesion, activating AKT. ROCK1 is a major downstream effector of RhoA and it is a regulator of the actomyosin cytoskeleton, influencing cell morphology, cell-cell and cell-matrix adhesion [55]. All these molecules are involved in the regulation of matrix stiffness. The latter influences cell migration, proliferation and survival [56, 57], and focal adhesions can form and grow only if they experience pulling forces through their cytoskeleton. It is known that growth on soft substrates leads to smaller focal adhesions, containing less phosphotyrosine and reduced cytoskeletal organisation [58]. In QUC, molecules associated to the cytoskeletal stiffness, such as vinculin and detyrosinated $\alpha$-tubulin, are decreased, suggesting that the mechanical properties of the epithelial barrier are changed and the stiffness reduced $[53,59]$. This reduction, described for the first time in QUC patients, could account also for an impaired cell renewal due to alteration of the mechanical properties of the tissue [57]. Further studies, specifically conducted on primary cultures are in progress and are aimed at precisely deciphering the role of membrane stiffness in invasion and cell renewal in IBDs. Interestingly, FAK and vimentin increased, whereas ROCK1, which is involved in actin cytoskeleton organization and remodelling, decreased, thus supporting the hypothesis of a decreased stiffness in QUC patients. Inflammation of the 
mucosa exacerbated both molecular changes, as pinpointed by the increase in COL6A1, TNC, ROCK1, vimentin and lymphocyte homing to intestinal mucosa, which was indicated by increased integrin $\beta 7$ and $\alpha 4$ levels; on the contrary, decreased levels of FAK were observed. Concerning detyrosinated $\alpha$-tubulin and vinculin, these molecules were not affected by the inflammatory process; their expression remained low, further suggesting the presence of constitutive alterations of the epithelial structure in UC. Indeed, changes in the epithelial barrier integrity may impact the autophagic process to eliminate damaged protein and organelles, and possible infiltrating bacteria. However, QUC patients are characterized by the activation of chaperone-mediated autophagy and macroautophagy. This conclusion is supported by many evidences at the molecular level, such as the metabolic stimulation of mTOR, thanks to increased isoleucine production, the activation of TORC promoting cell survival, the unchanged levels of HSC70, the increased levels of p62, MYH9 and MVP. All these molecules act in synergy and are harmonically regulated to sustain this process. In fact, the increase of beclin-1 was paralleled by the inhibitory action of Bcl-xL. Furthermore, LC3BII/LC3BI was down-regulated, indicating that LC3BI is not converted to LC3BII through lipidation. Collectively, these results suggest that the chaperone-mediated autophagy was not active and the cell adopted macroautophagy to eliminate waste material. Under inflammation, MVP, p62 and Bcl-xl remained up-regulated this indicates that macroautophagy is a characteristic trait of UC patients.

Given the role of autophagy in alleviating ER stress and in regulating immune response, further support to this process is provided by COPA. COPA is able to promote the binding of proteins targeted for retrograde Golgi to ER transport and its increase can counteract ER stress and upregulation of Th17 priming cytokines in QUC patients. Interestingly, it has been recently described that gene mutations are associated to arthritis and to the COPA syndrome [60].

At variance, a different picture characterized QCD, Keratin 8 and 18 were decreased, leading to increased sensitivity of the epithelium to stress conditions and to TNF-mediated apoptosis [45]. COL6A1 increased in ICD only, while TNC decreased, together with detyrosinated $\alpha$-tubulin and vinculin, both in quiescent and inflamed $\mathrm{CD}$, indicating that the latter are structural variations that are not influenced by the inflammatory process and are typical traits of IBDs. However, the decrease of TNC combined with vinculin and detyrosinated $\alpha$-tubulin have an impact on signalling inside the cell, thus inhibiting FAK and ROCK1 
expression in QCD and hindering cell adhesion and actin filament assembly. Also in this case, the signalling is influenced by an increase in TGF- $\beta$ and inflammation, which stimulate collagen VI expression, but it is unable to rescue FAK and ROCK 1 signalling. Collectively, these data suggest that some rescue mechanisms of mechanical membrane integrity are blunted in QCD patients, leaving them more exposed to bacterial invasion. Furthermore, because vimentin increased both in QCD and ICD, it could be associated to interstitial sub-epithelial fibroblasts-to-myofibroblasts transition and therefore contribute to fibrotic structures generation [34-36], which is a common complication of CD. To support this hypothesis, further experiments adopting primary cell cultures will be required.

Metabolic rewiring is another point characterising QCD, when it is finalised to sustain energy demand involving polyamine synthesis and citrate homeostasis maintenance. Furthermore, the increase in ERLIN2, which mediates the endoplasmic reticulum-associated degradation (ERAD) of inositol 1,4,5-trisphosphate receptors (IP3Rs) [61, 62] and of sterol-accelerated of HMGCR [63], indicates that the deregulation of cellular cholesterol homeostasis may promote ER retention of the SCAP-SREBP complex [64-66], thus highlighting the role of changes in cholesterol homeostasis in CD. Such changes have never been described before: interestingly, they may account for alterations of cell membrane, ER and Golgi apparatus' structure in IEC from CD.

Furthermore, the disease appears to be characterized by ER stress and UPR, since GRP78, GRP58, and PRDX2 are reduced.

Autophagic impairment represents another critical point in CD. This impairment has already been suggested, since ATG genes polymorphysms have been identified in $\mathrm{CD}$, though a detailed analysis at molecular level in patients is still lacking. Proteomics indicate a decrease in HSC70, which is a key factor regulating chaperone-mediated autophagy. This decrease implies the absence of the chaperone-mediated autophagy, which is an important process for the clearance of damaged cells and organelles. The analysis of AKT/mTOR pathways, shown as supporting information in Figure 4S, combined with the decrease of p62, MYH9 and MVP, the inhibition of beclin-1 by Bcl-xL and the down regulation of LC3BII/LC3BI, indicates that both micro and macroautophagy are impaired and inflammation does not induce significant changes to overcome this issue. According to the present results, it can be concluded that, not only autophagy is 
impaired in $\mathrm{CD}$ but several processes involved in protein degradation are also blunted. To counteract this severe authophagic impairment associated with energy deficiency, it could be relevant, at least for QCD patients, to sustain citrate production through polyamines supplementation, which can possibly ameliorate the CD phenotype.

In conclusion, this study shows that specific protein profiles are associated with UC and CD. The study also outlines the relevance of autophagy and epithelial cell integrity and identifies specific markers that are likely to characterise UC and CD in quiescent and inflammatory conditions. Furthermore, our study identifies the role of membrane stiffness in mechanotransduction signalling and its impact on cell death, metabolism and stress response. Finally, these results highlight the importance of unbiased proteomic approaches, which have been valuable tools in understanding the complex mechanisms associated to IBD. The authors hope these results help pave the way for more specific validations of dysregulated proteins in larger cohorts. 


\section{Acknowledgements}

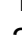

2

3

4

5

6

7

8

9

10

11

12

13

14

15

16

17

18

19

20

21

22

23

24

25

26

27

28

29

30

31

32

33

34

35

36

37

38

39

40

41

42

43

44

45

46

47

48

49

50

51

52

53

54

55

56

57

58

59

60
This work was supported by the Italian Ministry of Education, University and Research (MIUR), grant FIRB RBRN07BMCT and PRIN 2015FBNB5Y to CG.

All authors have no conflict of interest to declare. 


\section{References}

[1] Birchenough, G. M., Johansson, M. E., Gustafsson, J. K., Bergstrom, J. H., Hansson, G. C., New developments in goblet cell mucus secretion and function. Mucosal immunology 2015, 8, 712-719.

[2] Pastorelli, L., De Salvo, C., Mercado, J. R., Vecchi, M., Pizarro, T. T., Central role of the gut epithelial barrier in the pathogenesis of chronic intestinal inflammation: lessons learned from animal models and human genetics. Frontiers in immunology 2013, 4, 280.

[3] Das, K. M., Sakamaki, S., Vecchi, M., Ulcerative colitis: specific antibodies against a colonic epithelial Mr 40,000 protein. Immunological investigations 1989, 18, 459-472.

[4] Bianco, A. M., Girardelli, M., Tommasini, A., Genetics of inflammatory bowel disease from multifactorial to monogenic forms. World journal of gastroenterology 2015, 21, 12296-12310.

[5] McGovern, D. P., Kugathasan, S., Cho, J. H., Genetics of Inflammatory Bowel Diseases. Gastroenterology 2015, 149, 1163-1176 e1162.

[6] Hugot, J. P., Chamaillard, M., Zouali, H., Lesage, S., et al., Association of NOD2 leucine-rich repeat variants with susceptibility to Crohn's disease. Nature 2001, 411, 599-603.

[7] Ogura, Y., Bonen, D. K., Inohara, N., Nicolae, D. L., et al., A frameshift mutation in NOD2 associated with susceptibility to Crohn's disease. Nature 2001, 411, 603-606.

[8] Hampe, J., Franke, A., Rosenstiel, P., Till, A., et al., A genome-wide association scan of nonsynonymous SNPs identifies a susceptibility variant for Crohn disease in ATG16L1. Nature genetics 2007, 39, 207-211.

[9] Henckaerts, L., Cleynen, I., Brinar, M., John, J. M., et al., Genetic variation in the autophagy gene ULK1 and risk of Crohn's disease. Inflammatory bowel diseases 2011, 17, 1392-1397. 
[10] Freeman, E. B., Koglmeier, J., Martinez, A. E., Mellerio, J. E., et al., Gastrointestinal complications of epidermolysis bullosa in children. The British journal of dermatology 2008, 158, 1308-1314.

[11] Blaydon, D. C., Biancheri, P., Di, W. L., Plagnol, V., et al., Inflammatory skin and bowel disease linked to ADAM17 deletion. The New England journal of medicine 2011, 365, 1502-1508.

[12] Kern, J. S., Herz, C., Haan, E., Moore, D., et al., Chronic colitis due to an epithelial barrier defect: the role of kindlin-1 isoforms. The Journal of pathology 2007, 213, 462-470.

[13] Hsieh, S. Y., Shih, T. C., Yeh, C. Y., Lin, C. J., et al., Comparative proteomic studies on the pathogenesis of human ulcerative colitis. Proteomics 2006, 6, 5322-5331.

[14] Shkoda, A., Werner, T., Daniel, H., Gunckel, M., et al., Differential protein expression profile in the intestinal epithelium from patients with inflammatory bowel disease. Journal of proteome research 2007, 6, 1114-1125.

[15] Nanni, P., Mezzanotte, L., Roda, G., Caponi, A., et al., Differential proteomic analysis of HT29 Cl.16E and intestinal epithelial cells by LC ESI/QTOF mass spectrometry. Journal of proteomics 2009, 72, 865-873.

[16] Poulsen, N. A., Andersen, V., Moller, J. C., Moller, H. S., et al., Comparative analysis of inflamed and non-inflamed colon biopsies reveals strong proteomic inflammation profile in patients with ulcerative colitis. BMC gastroenterology 2012, 12, 76.

[17] Li, N., Wang, X., Zhang, Y., Zhai, J., et al., Comparative proteomics analysis of serum proteins in ulcerative colitis patients. Molecular biology reports 2012, 39, 5659-5667. 
[18] De Palma, S., Capitanio, D., Vasso, M., Braghetta, P., et al., Muscle proteomics reveals novel insights into the pathophysiological mechanisms of collagen VI myopathies. Journal of proteome research 2014, 13, 5022-5030.

[19] Giussani, M., De Maria, C., Vasso, M., Montemurro, F., Triulzi, T., Tagliabue, E., Gelfi, C., Vozzi, G., Biomimicking of the Breast Tumor Microenvironment. Current Molecular Biology Reports 2015, 1, 71-76.

[20] Pastorelli, L., Garg, R. R., Hoang, S. B., Spina, L., et al., Epithelial-derived IL-33 and its receptor ST2 are dysregulated in ulcerative colitis and in experimental Th1/Th2 driven enteritis. Proceedings of the National Academy of Sciences of the United States of America 2010, 107, 8017-8022.

[21] Taylor, C. F., Paton, N. W., Lilley, K. S., Binz, P. A., et al., The minimum information about a proteomics experiment (MIAPE). Nature biotechnology 2007, 25, 887-893.

[22] Moriggi, M., Vasso, M., Fania, C., Capitanio, D., et al., Long term bed rest with and without vibration exercise countermeasures: effects on human muscle protein dysregulation. Proteomics 2010, 10, 3756-3774.

[23] Marouga, R., David, S., Hawkins, E., The development of the DIGE system: 2D fluorescence difference gel analysis technology. Analytical and bioanalytical chemistry 2005, 382, 669-678.

[24] Viswanathan, S., Unlu, M., Minden, J. S., Two-dimensional difference gel electrophoresis. Nature protocols 2006, 1, 1351-1358.

[25] Benjamini, Y., Hochberg, Y., On the adaptive control of the false discovery fate in multiple testing with independent statistics. J Educ Behav Stat 2000, 25, 60-83. 
[26] Fania, C., Anastasia, L., Vasso, M., Papini, N., et al., Proteomic signature of reversine-treated murine fibroblasts by 2-D difference gel electrophoresis and MS: possible associations with cell signalling networks. Electrophoresis 2009, 30, 2193-2206.

[27] Wang, X., Chen, P., Cui, J., Yang, C., Du, H., Keratin 8 is a novel autoantigen of rheumatoid arthritis. Biochemical and biophysical research communications 2015, 465, 665-669.

[28] He, X. Y., Yang, Y. X., Yang, S. Y., Changes of the HSD17B10 gene expression levels in ulcerative colitis. Inflammatory bowel diseases 2013, 19, E23-24.

[29] Hedl, M., Lahiri, A., Ning, K., Cho, J. H., Abraham, C., Pattern recognition receptor signaling in human dendritic cells is enhanced by ICOS ligand and modulated by the Crohn's disease ICOSLG risk allele. Immunity 2014, 40, 734-746.

[30] Di Blasi, C., Sansanelli, S., Ruggieri, A., Moriggi, M., et al., A CASQ1 founder mutation in three Italian families with protein aggregate myopathy and hyperCKaemia. Journal of medical genetics 2015, 52, 617-626.

[31] Ruegg, C., Postigo, A. A., Sikorski, E. E., Butcher, E. C., et al., Role of integrin alpha 4 beta 7/alpha 4 beta $\mathrm{P}$ in lymphocyte adherence to fibronectin and VCAM-1 and in homotypic cell clustering. The Journal of cell biology 1992, 117, 179-189.

[32] Gorfu, G., Rivera-Nieves, J., Ley, K., Role of beta7 integrins in intestinal lymphocyte homing and retention. Current molecular medicine 2009, 9, 836-850.

[33] Dart, R. J., Samaan, M. A., Powell, N., Irving, P. M., Vedolizumab: toward a personalized therapy paradigm for people with ulcerative colitis. Clinical and experimental gastroenterology 2017, 10, 57-66. 
[34] Bataille, F., Rohrmeier, C., Bates, R., Weber, A., et al., Evidence for a role of epithelial mesenchymal transition during pathogenesis of fistulae in Crohn's disease. Inflammatory bowel diseases 2008, 14, 15141527.

[35] Gerarduzzi, C., Di Battista, J. A., Myofibroblast repair mechanisms post-inflammatory response: a fibrotic perspective. Inflammation research : official journal of the European Histamine Research Society ... [et al.] 2017, 66, 451-465.

[36] Mor-Vaknin, N., Legendre, M., Yu, Y., Serezani, C. H., et al., Murine colitis is mediated by vimentin. Scientific reports 2013, 3, 1045.

[37] Dubuquoy, L., Jansson, E. A., Deeb, S., Rakotobe, S., et al., Impaired expression of peroxisome proliferator-activated receptor gamma in ulcerative colitis. Gastroenterology 2003, 124, 1265-1276.

[38] Zhou, X., Cao, L., Jiang, C., Xie, Y., et al., PPARalpha-UGT axis activation represses intestinal FXRFGF15 feedback signalling and exacerbates experimental colitis. Nature communications 2014, 5, 4573.

[39] Levett, D. Z., Vigano, A., Capitanio, D., Vasso, M., et al., Changes in muscle proteomics in the course of the Caudwell Research Expedition to Mt. Everest. Proteomics 2015, 15, 160-171.

[40] De Palma, S., Leone, R., Grumati, P., Vasso, M., et al., Changes in muscle cell metabolism and mechanotransduction are associated with myopathic phenotype in a mouse model of collagen VI deficiency. PloS one 2013, 8, e56716.

[41] Zhao, B., Qi, Z., Li, Y., Wang, C., et al., The non-muscle-myosin-II heavy chain Myh9 mediates colitis-induced epithelium injury by restricting Lgr5+ stem cells. Nature communications 2015, 6, 7166. 
[42] Shults, N. V., Das, D., Suzuki, Y. J., Major vault protein in cardiac and smooth muscle. Receptors \& clinical investigation 2016, 3 .

[43] Iida, T., Onodera, K., Nakase, H., Role of autophagy in the pathogenesis of inflammatory bowel disease. World journal of gastroenterology 2017, 23, 1944-1953.

[44] Dotti, I., Mora-Buch, R., Ferrer-Picon, E., Planell, N., et al., Alterations in the epithelial stem cell compartment could contribute to permanent changes in the mucosa of patients with ulcerative colitis. Gut 2016.

[45] Owens, D. W., Wilson, N. J., Hill, A. J., Rugg, E. L., et al., Human keratin 8 mutations that disturb filament assembly observed in inflammatory bowel disease patients. Journal of cell science 2004, 117, 1989-1999.

[46] Roediger, W. E., The colonic epithelium in ulcerative colitis: an energy-deficiency disease? Lancet $1980,2,712-715$.

[47] Steinhart, A. H., Brzezinski, A., Baker, J. P., Treatment of refractory ulcerative proctosigmoiditis with butyrate enemas. The American journal of gastroenterology 1994, 89, 179-183.

[48] Patz, J., Jacobsohn, W. Z., Gottschalk-Sabag, S., Zeides, S., Braverman, D. Z., Treatment of refractory distal ulcerative colitis with short chain fatty acid enemas. The American journal of gastroenterology 1996, $91,731-734$.

[49] Cescon, M., Gattazzo, F., Chen, P., Bonaldo, P., Collagen VI at a glance. Journal of cell science 2015, $128,3525-3531$. 
[50] Sabatelli, P., Gualandi, F., Gara, S. K., Grumati, P., et al., Expression of collagen VI alpha5 and alpha6 chains in human muscle and in Duchenne muscular dystrophy-related muscle fibrosis. Matrix biology : journal of the International Society for Matrix Biology 2012, 31, 187-196.

[51] Neumann, C., Yu, A., Welge-Lussen, U., Lutjen-Drecoll, E., Birke, M., The effect of TGF-beta2 on elastin, type VI collagen, and components of the proteolytic degradation system in human optic nerve astrocytes. Investigative ophthalmology \& visual science 2008, 49, 1464-1472.

[52] Tadokoro, S., Shattil, S. J., Eto, K., Tai, V., et al., Talin binding to integrin beta tails: a final common step in integrin activation. Science 2003, 302, 103-106.

[53] Omachi, T., Ichikawa, T., Kimura, Y., Ueda, K., Kioka, N., Vinculin association with actin cytoskeleton is necessary for stiffness-dependent regulation of vinculin behavior. PloS one 2017, 12, $\mathrm{e} 0175324$.

[54] Jaffe, A. B., Hall, A., Rho GTPases: biochemistry and biology. Annual review of cell and developmental biology 2005, 21, 247-269.

[55] Rath, N., Olson, M. F., Rho-associated kinases in tumorigenesis: re-considering ROCK inhibition for cancer therapy. EMBO reports 2012, 13, 900-908.

[56] Wells, R. G., The role of matrix stiffness in regulating cell behavior. Hepatology 2008, 47, 1394-1400.

[57] Urciuolo, A., Quarta, M., Morbidoni, V., Gattazzo, F., et al., Collagen VI regulates satellite cell selfrenewal and muscle regeneration. Nature communications 2013, 4, 1964.

[58] Pelham, R. J., Jr., Wang, Y., Cell locomotion and focal adhesions are regulated by substrate flexibility. Proceedings of the National Academy of Sciences of the United States of America 1997, 94, 13661-13665. 32 Wiley - VCH 
[59] Kerr, J. P., Robison, P., Shi, G., Bogush, A. I., et al., Detyrosinated microtubules modulate mechanotransduction in heart and skeletal muscle. Nature communications 2015, 6, 8526.

[60] Watkin, L. B., Jessen, B., Wiszniewski, W., Vece, T. J., et al., COPA mutations impair ER-Golgi transport and cause hereditary autoimmune-mediated lung disease and arthritis. Nature genetics 2015, 47, 654-660.

[61] Pearce, M. M., Wormer, D. B., Wilkens, S., Wojcikiewicz, R. J., An endoplasmic reticulum (ER) membrane complex composed of SPFH1 and SPFH2 mediates the ER-associated degradation of inositol 1,4,5-trisphosphate receptors. The Journal of biological chemistry 2009, 284, 10433-10445.

[62] Pearce, M. M., Wang, Y., Kelley, G. G., Wojcikiewicz, R. J., SPFH2 mediates the endoplasmic reticulum-associated degradation of inositol 1,4,5-trisphosphate receptors and other substrates in mammalian cells. The Journal of biological chemistry 2007, 282, 20104-20115.

[63] Jo, Y., Sguigna, P. V., DeBose-Boyd, R. A., Membrane-associated ubiquitin ligase complex containing gp78 mediates sterol-accelerated degradation of 3-hydroxy-3-methylglutaryl-coenzyme A reductase. The Journal of biological chemistry 2011, 286, 15022-15031.

[64] Huber, M. D., Vesely, P. W., Datta, K., Gerace, L., Erlins restrict SREBP activation in the ER and regulate cellular cholesterol homeostasis. The Journal of cell biology 2013, 203, 427-436.

[65] Tsai, Y. C., Leichner, G. S., Pearce, M. M., Wilson, G. L., et al., Differential regulation of HMG-CoA reductase and Insig-1 by enzymes of the ubiquitin-proteasome system. Molecular biology of the cell 2012, $23,4484-4494$.

[66] Goldstein, J. L., DeBose-Boyd, R. A., Brown, M. S., Protein sensors for membrane sterols. Cell 2006, $124,35-46$. 


\section{Legends}

Figure 1. Proteomic analysis by 2D-DIGE. Histograms of differentially expressed proteins observed by 2DDIGE in non-inflamed UC (QUC) and CD (QCD) versus controls (C). Two different comparisons were performed: QUC vs C (gray bars) and QCD vs C (black bars). Statistical analysis was performed using DeCyder EDA 1.0 module; independent one-way ANOVA coupled to Turkey's multiple group comparison test $(\mathrm{P}<0.01)$ were used to determine protein spots significantly different between the analyses.

Differentially expressed proteins are indicated by their gene name and they are expressed as \% of fold change. List of proteins differentially expressed in all comparisons were grouped according to their role together with statistical analysis and are presented as raw data sets in Supplementary Tables 2S. Cytoskeleton organization: KRT8, Keratin type II cytoskeletal 8; KRT18, Keratin type I cytoskeletal 18; KRT19, Keratin type I cytoskeletal 19; ACTB, Actin cytoplasmic 1; TUBB4B, Tubulin beta-2C chain; IMMT, Mitochondrial inner membrane protein. Metabolic proteins: GPD2, Glycerol-3-phosphate dehydrogenase mitochondrial; PDHB, Pyruvate dehydrogenase E1 component subunit beta mitochondrial precursor; DLD, Dihydrolipoyl dehydrogenase mitochondrial; SDHA, Succinate dehydrogenase [ubiquinone] flavoprotein subunit mitochondrial; NDUFS3, NADH dehydrogenase [ubiquinone] iron-sulfur protein 3 mitochondrial; ATP5B, ATP synthase subunit beta; ACADSB, Short/branched chain specific acylCoA dehydrogenase mitochondrial; HSD17B10, 3-hydroxyacyl-CoA dehydrogenase type-2; ALDH9A1, 4trimethylaminobutyraldehyde dehydrogenase. Stress and antioxidant proteins: HSP60, $60 \mathrm{kDa}$ heat shock protein mitochondrial; HSC70, Heat shock cognate $71 \mathrm{kDa}$ protein; HSPA1A/B, Heat shock $70 \mathrm{kDa}$ protein 1A/1B; GRP78, 78 kDa glucose-regulated protein (GRP78); GRP58, Protein disulfide-isomerase A3; PRDX2, Peroxiredoxin-2. DNA synthesis: PHB, Prohibitin. RNA processing: LGALS3, Galectin-3. Protein biosynthesis: TUFM, Elongation factor tu mitochondrial. Transport proteins: VDAC1, Voltage-dependent anion-selective channel protein 1; MRPS22, 28S ribosomal protein S22 mitochondrial; ERLIN2, Endoplasmic reticulum lipid raft-associated protein 2. Others proteins: ANXA4, Annexin A4; ANXA5, Annexin A5; CA1, Carbonic anhydrase 1; RACK1, Receptor of activated protein C kinase 1; C1QBP, Complement component 1 Q subcomponent-binding protein, mitochondrial. 
Figure 2. Proteomic analysis by Isotope-Coded Protein Labeling (ICPL). Histograms of differentially expressed proteins observed by ICPL in non-inflamed UC (QUC) and CD (QCD) versus controls (C). Two different comparisons were performed: QUC vs C (gray bars) and QCD vs C (black bars). Differentially expressed proteins are indicated by their gene name and they are expressed as $\%$ of fold change. List of proteins differentially expressed in all comparisons were grouped according to their role together with statistical analysis and are presented as raw data sets in Supplementary Tables 3S. Cytoskeleton organization: MYH9, Myosin-9; KRT10, Keratin type I cytoskeletal 10; KRT14, Keratin type I cytoskeletal 14; KRT18, Keratin type I cytoskeletal 18; TUBA1B, Tubulin alpha-ubiquitous chain; VIM, Vimentin. Metabolic protein: HSD11B2, Corticosteroid 11-beta-dehydrogenase isozyme 2. RNA splicing and transport: HNRNPA1, Heterogeneous nuclear ribonucleoprotein A1; MVP, Major vault protein. Chromatin organization: HIST2H2AA3, Histone H2A type 2-A; HIST1H2AB, Histone H2A type 1-B; HIST1H2AC, Histone H2A type 1-C; HIST1H3A, Histone H3.1; HIST1H4A, Histone H4; SFPQ, Splicing factor prolineand glutamine-rich. Protein folding: TRAP1, Heat shock protein $75 \mathrm{kDa}$ mitochondrial precursor; PPIB, Peptidyl-prolyl cis-trans isomerase B precursor. Transport proteins: RRBP1, Ribosome-binding protein 1; COPA, Coatomer subunit alpha; ATP1A1, Sodium/potassium-transporting ATPase alpha-1 chain precursor. Figure 3. Stiffness and mechanotransduction. Representative immunoblot images (A, C) and histograms (means $\pm \mathrm{SD}$; B, D) of proteins expression of collagen type VI Alpha 1 Chain (COL6A1), tenascin C (TNC), Integrin $\beta 7$, Integrin $\alpha 4$, focal adhesion kinase (FAK), Rho-associated protein kinase 1 (ROCK1), Vinculin, detyrosinated $\alpha$-Tubulin (de Tyr $\alpha$-Tubulin) and Vimentin, in inflamed and quiescent ulcerative colitis (IUC, QUC) compared to controls (C), and in inflamed and quiescent Crohn's Disease (ICD, QCD) compared to C. The data were normalized against the total amount of loaded proteins stained with Sypro Ruby. Statistical analysis was performed by Student's t-test $(\mathrm{p}<0.05)$ * change compared to control; $\$$ variation between QUC vs IUC and QCD vs ICD.

Figure 4. Upstream regulators of fatty acid metabolism. Representative immunoblot images (A, C) and histograms (means $\pm \mathrm{SD} ; \mathrm{B}, \mathrm{D})$ of proteins expression of citrate synthase, fatty acid synthase (FASN), peroxisome proliferator-activated receptors gamma (ppary) and peroxisome proliferator-activated receptors alpha (ppar $\alpha$ ), in inflamed and quiescent ulcerative colitis (IUC, QUC) compared to controls (C), and in 
inflamed and quiescent Crohn's Disease (ICD, QCD) compared to C. The data were normalized against the total amount of loaded proteins stained with Sypro Ruby. Statistical analysis was performed by Student's ttest $(\mathrm{p}<0.05)$ * change compared to control; $\$$ variation between QUC vs IUC and QCD vs ICD.

Figure 5. Autophagy. Representative immunoblot images (C, E, G) and histograms (means $\pm \mathrm{SD} ; \mathrm{A}, \mathrm{B}, \mathrm{D}$, F) of proteins expression of Heat shock cognate $71 \mathrm{kDa}$ protein (HSC70), myosin heavy chain 9 (MYH9), major vault protein (MVP), ubiquitin-binding protein p62 (p62), light chain 3B (LC3B), bcl-2-like protein 4 (Bax), beclin1, B-cell lymphoma-extra large (Bcl-xL), BCL2/adenovirus E1B 19 kDa protein-interacting protein 3 (Bnip3) and B-cell leukemia/lymphoma 2 (Bcl-2), in inflamed and quiescent ulcerative colitis (IUC, QUC) compared to controls (C), and in inflamed and quiescent Crohn's Disease (ICD, QCD) compared to C. The data were normalized against the total amount of loaded proteins stained with Sypro Ruby. Statistical analysis was performed by Student's t-test $(\mathrm{p}<0.05)$ * change compared to control; $\$$ variation between QUC vs IUC and QCD vs ICD. 
Page 37 of 41

1 Cytoskeleton

2 organization

4
5
6
7
8
9

10 Metabolic

11 proteins

12

13

14

15

16

17

18

19

20 Stress and

21 antioxidant

22 proteins

23

24

25

$\mathrm{R} \mathbb{2}$

Prôtein biosynthesis

29

30

31

32

33

34

35

36

37

38

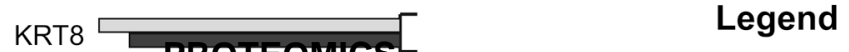

KRT8

KRT8

KRT18

KRT18

KRT19

ACTB

TUBB4B

IMMT

GPD2

PDHB

PDHB

DLD

SDHA

NDUFS3

ATP5B

ATP5B

ATP5B

ACADSB

HSD17B10

ALDH9A1

HSP60

HSC70

HSC70

HSPA1A/B

GRP78

GRP58

PRDX2

$\mathrm{PHB}$

LGALS3

LGALS3

LGALS3

TUFM

VDAC1

MRPS22

ERLIN2

ANXA4

ANXA5

CA1

RACK1

C1QBP $-80$

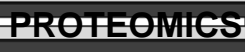

QUC vs C QCD vs C 


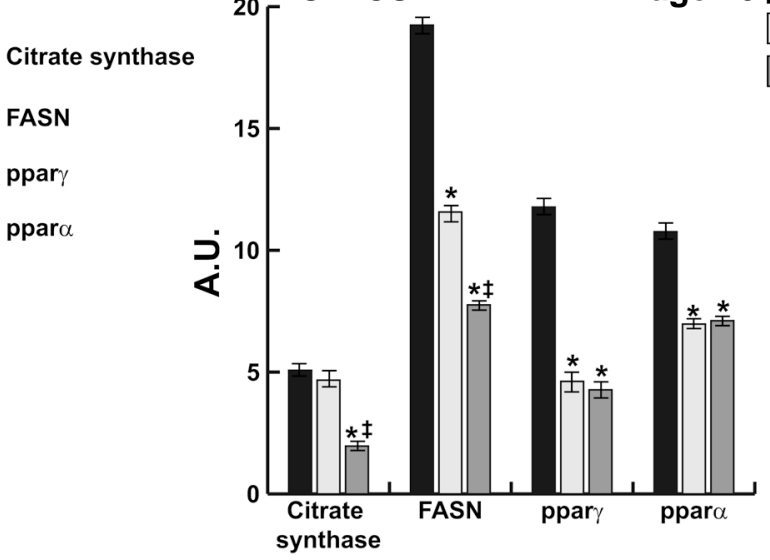

12

5

6

7

8

9

10

11

13

14

D

165 QCD ICD

17

18

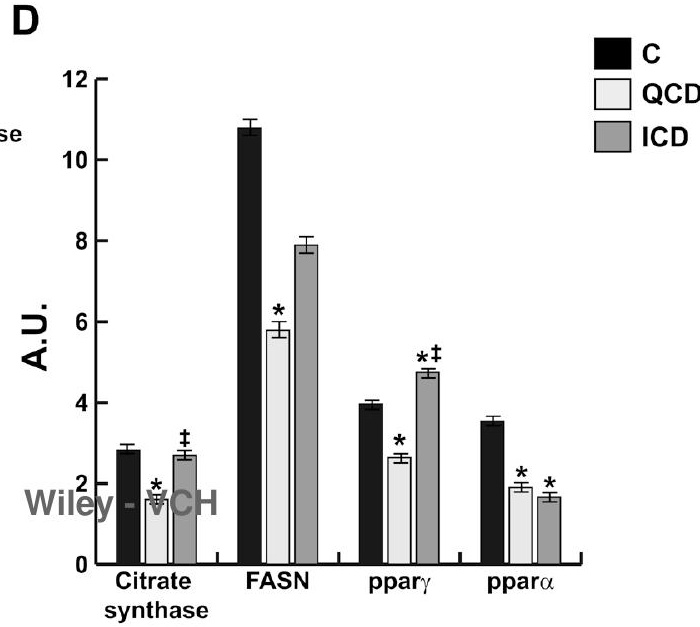

$C$

Citrat
FASN

ppar $\gamma$

ppar $\alpha$

21

22

23

24

25

26

27

28 


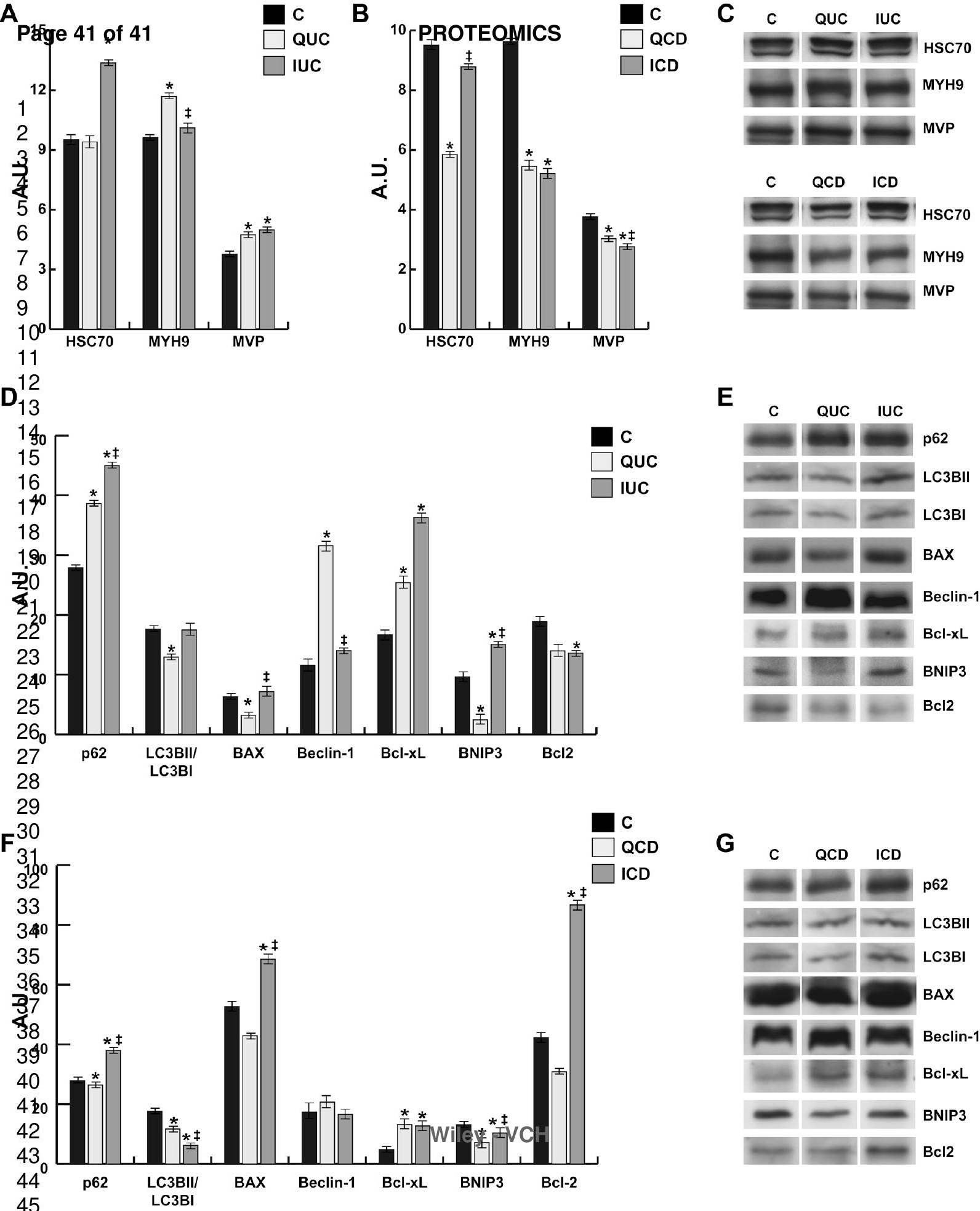

